\title{
Computer Assisted Alignment of the Oxford Unicompartmental Knee Arthroplasty: The Kingston Experience with Three Techniques
}

\author{
D.J. Mayman ${ }^{1}$, J.F. Rudan ${ }^{1}$, D.R. Pichora ${ }^{1}$, D. Watson ${ }^{1}$, and R.E. Ellis ${ }^{1,2}$ \\ 1 Division of Orthopædics, Department of Surgery \\ ${ }^{2}$ School of Computing \\ Queen's University at Kingston, Canada K7L 3N6
}

\begin{abstract}
Accuracy and precision of inserting joint implants is essential for successful long-term prosthetic function. Clinical and radiographic assessment of Oxford unicompartmental knee arthroplasty using 3 computer-assisted techniques (CT based, "morphing" and fluoro-based) was undertaken on 17 patients. They were followed for at least one year postoperatively. Clinical outcome assessments: Knee Society Scores, WOMAC and SF-36 all indicated good to excellent results. All methods worked well with the manufacture tolerance of $10^{\circ}$ in both planes. However, CT based and fluoro-based techniques were significantly better than the morphing technique.
\end{abstract}

\section{Objective}

The objective of this project was to review three computer-assisted techniques for inserting the Oxford (Biomet, Merck, USA) unicompartmental knee arthroplasties. CT based, fluoro-based and "morphing" techniques were compared with regards to accuracy and variability of component positioning.

\section{Background}

Oxford unicompartmental arthroplasties have been performed with good results for more than 10 years [2]. Instruments and techniques have evolved over the years. Standard insertion technique describes the use of a femoral intramedullary guide for femoral component positioning [1]. Inaccuracies have been associated with use of the standard intramedullary guide [4,3].

\section{Materials and Methods}

For the last year we have been using three computer-assisted techniques for insertion of the Oxford Unicompartmental knee arthroplasty. Initially we used a CT based platform. Subsequently, as new technology has evolved, we have used morphing technology and a fluoro-based platform to obtain accurate alignment. Patients were all seen in a preadmission clinic where pre-operative SF-36, WOMAC scores, and Knee Society Scores 
were obtained. All patients had standing AP and lateral knee X-rays as well as skyline X-rays. Patients being operated on with the CT based system had pre operative CT scans. Intraoperatively, a dynamic reference base was fixed to the patients femur and the chosen computer-assisted technique was used to direct the rotation of the tibial cut as well as the alignment of the femoral cutting jig. The remainder of the procedure was carried out as described in the surgical technique guide [1]. Postoperatively full length AP and lateral femur X-rays were completed in order to measure alignment of the femoral component. Patients are being followed post-operatively with SF-36, WOMAC, Knee Society Scores, and X-rays.

\section{Results}

To date we have completed 17 computer-assisted Oxford Unicompartmental Knee Arthroplasties. The average error in the AP and lateral planes using the CT based system was $3.2^{\circ}$ and $2.1^{\circ}$ respectively. Errors using morphing techniques were unacceptably high and the technique was abandoned clinically when we developed the ability to use a fluoro-based system. The average error in the AP plane using the fluoro-based system was 2.2 degrees. The average error in the lateral plane using the fluoro-based system was $1.3^{\circ}$.

\section{Conclusions}

Computer-assisted techniques are allowing us to perform minimally invasive arthroplasty procedures with great accuracy. Currently we are able to use either a CT based system or a fluoro-based system to accurately insert the femoral component of the Oxford Unicompartmental Knee arthroplasty. At this time we are not using "morphing" techniques at our center but we are continuing to work on refinement of the technology.

Acknowledgments. This research was supported in part by the Institute for Robotics and Intelligent Systems, the Ontario Research and Development Challenge Fund, and the Natural Sciences and Engineering Research Council of Canada.

\section{References}

1. Merck Biomet. Surgical technique guide: Oxford unicompartmental arthroplasty phase 3, 2002.

2. D. W. Murray and J. W. Goodfellow an J. J. O'Connor. The oxford medial unicompartmental arthroplasty: A ten-year survival study. Journal of Bone and Joint Surgery, 80(6):983-989, 1998.

3. J. Novotny, M. H. Gonzalez, F. M. L. Amirouche, and Y. C. Li. Geometric analysis of potential error in using femoral intramedullary guides in total knee arthroplasty. Journal of Arthroplasty, 12:641, 2001.

4. S. C. Reed and J. Gollish. The accuracy of femoral intramedullary guides in total knee arthroplasty. The Journal of Arthroplasty, 12(6):677-682, 1997. 